\title{
Daten teilen für die Forschung: Einstellungen und Perspektiven zur Datenspende in Deutschland
}

\author{
Wiebke Lesch, Gesine Richter und Sebastian C. Semler
}

\section{$1 \quad$ Hintergrund und Einleitung}

\subsection{Fragmentierte Datenbestände behindern Forschungsdatennutzung}

Medizinische Daten fallen an vielen Stellen im Gesundheitswesen an. Sie entstehen in Krankenhäusern, Arztpraxen, bei Heil- und Pflegediensten, Psychologen, Apotheken, Krankenkassen und privaten Versicherungen oder werden durch Patienten selbst erfasst. Könnte man diese Daten für die medizinische Forschung nutzen - auch Sekundärdatennutzung genannt - wäre damit ein immenser Erkenntnisgewinn zur Entwicklung neuer diagnostischer und therapeutischer Möglichkeiten verbunden. Mithilfe der Sekundärdatennutzung könnte beispielsweise in der klinischen Forschung die Machbarkeit von Studien besser bewertet werden, indem Rückschlüsse darüber gezogen werden, ob zu bestimmten Krankheitsbildern ausreichend viele Studienteilnehmer verfügbar sind. Potenziell geeignete Patienten könnten dann kontaktiert werden. Für die epidemiologische Forschung könnten im Sinne von Big Data Versorgungsdaten nach Trends, Mustern und Risikofaktoren untersucht werden und auf dieser Basis gezielt epidemiologische Studien

W. Lesch $(\bowtie) \cdot$ S. C. Semler

Technologie- und Methodenplattform für die vernetzte medizinische Forschung e. V., Berlin, Deutschland

E-Mail: Wiebke.Lesch@tmf-ev.de

S. C. Semler

E-Mail: Sebastian.Semler@tmf-ev.de

G. Richter

Institut für Experimentelle Medizin, Universität Kiel, 24105 Kiel, Deutschland

E-Mail: gesine.richter@iem.uni-kiel.de

G. Richter et al. (Hrsg.), Datenreiche Medizin und das Problem der Einwilligung, 
aufgesetzt werden, die diese Thesen evaluieren. Auch die Versorgungsforschung würde von der Sekundärdatennutzung profitieren. Sie nutzt reale Daten aus der medizinischen Versorgung, um Prozesse, Dienstleistungen und Richtlinien der Krankenversorgung zu bewerten. Obwohl eine Vielzahl verschiedener Datenquellen aus dem ersten und zweiten Gesundheitsmarkt existieren, die für die wissenschaftliche Sekundärdatennutzung in der klinischen und epidemiologischen Forschung sowie für die Versorgungsforschung von herausragendem Nutzen sein würden, liegen die Daten zu wenig strukturiert, zu wenig standardisiert und vor allem viel zu fragmentiert vor. Unterschiedliche Zuständigkeiten, z. B. in den verschiedenen Sektoren des Gesundheitswesens und zwischen Bund und Ländern, und ein fragmentierter Rechtsrahmen behindern einen durchgängigen Blick auf Patientenverläufe, Versorgungsprozesse und Meldedaten. So können patientenbezogene Daten aus technischen und aus datenschutzrechtlichen Gründen nicht über die Grenzen von Standorten hinweg verknüpft werden. Eine wichtige Aufgabe in Deutschland besteht deshalb darin, zeitnah die rechtlichen und infrastrukturellen Voraussetzungen für die wissenschaftliche Nutzung möglichst vieler medizinischer Datenbestände $\mathrm{zu}$ schaffen. Die Innovationsoffensive „Daten für Gesundheit“ der drei Bundesministerien für Gesundheit (BMG), Wirtschaft (BMWI) sowie Bildung und Forschung (BMBF) sieht deshalb in seiner Roadmap als prioritäre Handlungsfelder unter anderem den Strukturausbau zur digitalen Vernetzung der Gesundheitsforschung, die Erhöhung der Datenverfügbarkeit und der Datenqualität, die bessere Datenverknüpfung mit höheren Sicherheitsstandards sowie die Entwicklung klarer Anwendungsperspektiven für die Gesundheitsdaten (Bundesministerium für Bildung und Forschung 2020). Dies schließt insbesondere die Verknüpfung der Daten ein, welche aktuell durch datenschutzrechtliche Hürden erschwert bzw. verhindert wird. Gerade die Verknüpfung der Daten aus unterschiedlichen Datenbeständen birgt das Potenzial, wissenschaftlichen Wert zu schaffen.

\subsection{Initiativen zur Datenintegration}

\subsubsection{Deutschland}

Die Medizininformatik-Initiative (MII) schafft gegenwärtig in Deutschland die Voraussetzungen dafür, Routinedaten der klinischen Versorgung deutschlandweit für die medizinische Forschung verfügbar zu machen (Medizininformatik-Initiative 2020, Semler et al. 2018). Ziel der vom Bundesministerium für Bildung und Forschung geförderten Initiative ist die Verbesserung von Forschungsmöglichkeiten und der Patientenversorgung durch innovative IT-Lösungen. Diese sollen den Austausch und die Nutzung von Daten aus Krankenversorgung, klinischer und biomedizinischer Forschung über die Grenzen von Institutionen und Standorten hinweg ermöglichen. In mehreren großen Konsortien arbeiten alle Einrichtungen der Universitätsmedizin in Deutschland an über 30 Standorten gemeinsam mit Forschungseinrichtungen, Unternehmen, Krankenkassen und Patientenvertretern zusammen, um die Rahmenbedingungen für diesen Datenaustausch zu schaffen. Erkenntnisse aus der Forschung sollen somit 
direkt den Patienten zu Gute kommen. Die Gesundheitsdaten der Bürger sind für die medizinische Forschung von großem Wert. Um das Potenzial dieser Daten für die Gesundheitsforschung ausschöpfen zu können, bedarf es einerseits weitreichender Investitionen in die Verfügbarkeit, Verknüpfbarkeit und Verwertbarkeit dieser Daten. Andererseits ist ein entsprechender rechtlicher und ethisch vertretbarer Rahmen notwendig bezüglich der sogenannten Governance, d. h. eine Regelung wer was mit den Daten tun darf und wer darüber entscheidet. Datenschutz und Datensicherheit haben dabei höchste Priorität.

\subsubsection{Internationale Ansätze}

International wurden in verschiedenen Ländern Datenintegrationsinitiativen gestartet, von denen hier beispielhaft Australien und Finnland vorgestellt werden.

\section{Australien}

2018 wurde in Australien die digitale Gesundheitsakte „My Health Records“ von der australischen Regierung eingeführt (Australian Government 2020). MyHealthRecord ermöglicht es Patienten, unter eigener Kontrolle wichtige Gesundheitsinformationen mit Ärzten, Krankenhäusern und anderen Gesundheitsdienstleistern zu teilen (Deloitte 2016). MyHealthRecord ersetzt nicht die Krankenakte, sondern ergänzt sie in Form eines online verfügbaren Datenbestands z. B. zu Allergien, Medikation, Vorerkrankungen und Labortestergebnissen. Daten aus MyHealthRecord sollen ab 2020 für Forschungszwecke pseudonymisiert herausgegeben werden, nachdem die Arbeiten zur Schaffung der dafür notwendigen, technischen und organisatorischen Voraussetzung, insbesondere hinsichtlich des Datenschutzes und der Governance, abgeschlossen sind. Die Patienten werden der Forschungsnutzung ihrer Daten widersprechen können.

\section{Finnland}

Mit dem am 1. Mai 2019 in Finnland in Kraft getretenen Gesetz zur Sekundärdatennutzung wird ab dem Jahr 2020 FINDATA als „One-Stop-Agency“ einen Forschungszugang zu Sozial- und Gesundheitsdaten für Forschende ermöglichen (Ministry of Social Affairs and Health 2019). FINDATA ist als zentrale nationale Behörde für die Verwaltung und Herausgabe von Sozial- und Gesundheitsdaten der finnischen Bevölkerung gegründet worden und berät hinsichtlich der Datenverfügbarkeit, stellt zentral elektronische Erlaubnisse bereit und ermöglicht in einer geschützten virtuellen Umgebung die pseudonymisierte Datennutzung. Hierzu bedient FINDATA sich interoperabel vernetzter Datenbanken der einzelnen Datenhalter. Die Datenhoheit liegt dabei im Rahmen der nationalen Gesetzgebung bei den öffentlich-rechtlichen Datenhaltern. FINDATA wird dem Ministerium für Soziales und Gesundheit (Ministry of Social Affairs and Health) unterstellt und eine eigenständige juristische Person sein, die Teil des Nationalen Instituts für Gesundheit und Soziales (National Institute of Health and Welfare) ist. 
Die Intention hinter FINDATA ist es, dass Europa im Wettbewerb mit den USA und Asien gefordert ist, einen eigenen Forschungsdatenraum zu schaffen, dessen Stärke darin besteht, dass er vom berechtigten Vertrauen der Bürgerinnen und Bürger lebt. Hierzu bedarf es in enger Abstimmung mit dem privaten Sektor der Entwicklung einer Europäischen Leitlinie für die ,faire“ Datennutzung basierend auf den sogenannten FAIR-Prinzipien (Wilkinson et al. 2016, Europäische Kommission 2020). Ein solches Vertrauenslabel für faire Datennutzungen wünschen sich laut einer Umfrage von SITRA zufolge auch $63 \%$ der Deutschen (Parikka 2019).

\section{$2 \quad$ Einwilligung in die Forschungsdatenspende}

Um vorhandene medizinische Datenbestände in Deutschland überhaupt nachnutzen $\mathrm{zu}$ können, müssen zunächst die rechtlichen und infrastrukturellen Voraussetzungen betrachtet werden. Der verfassungsrechtliche Rahmen verspricht einerseits das Recht auf informationelle Selbstbestimmung (GG Art. 2 Abs. 1), gleichzeitig garantiert er auch die Wissenschaftsfreiheit (GG Art. 5 Abs. 3). Darüber hinaus reguliert ein europaweit einheitlicher Rechtsrahmen für die Verarbeitung personenbezogener medizinischer Daten die Forschungsdatennutzung. Nach Artikel 6 der allgemeinen EU-Datenschutzverordnung (Verordnung 2016/679 EU, EU-GDPR), welche im Mai 2018 in Kraft trat, ist die Verarbeitung personenbezogener medizinischer Daten nur erlaubt, wenn die betroffene Person in die Verarbeitung eingewilligt hat oder die Verarbeitung (d. h. die Forschung) im öffentlichen Interesse ist, sowie unter Bedingungen erfolgt, die im Recht der EU oder der Mitgliedstaaten festgelegt sind. Eines der wichtigsten Ziele der EUGDPR ist es, den Bürgern Kontrolle über ihre Daten zu gewähren, die im Forschungskontext insbesondere durch die Erteilung ihres Einverständnisses Ausübung findet.

In Deutschland sind die gesetzlichen Regeln fragmentiert, was die Rechtslage unüberschaubar und ihre praktische Umsetzung schwierig macht. Sämtliche Regeln beinhalten jedoch, dass eine Datennutzung ohne Einwilligung der Betroffenen nur im Ausnahmefall und auf der Grundlage einer Güterabwägung erlaubt ist (Strech et al. 2020).

Eine Datennutzung muss im Regelfall also durch eine informierte Einwilligung der betroffenen Personen legitimiert sein, d. h. sie müssen im Voraus über den Zweck, die Art der Nutzung, die Risiken und den Nutzen der betreffenden Studie informiert werden und in der Lage sein, über ihre Teilnahme freiwillig und ohne Zwang zu entscheiden. Nun liegt es häufig in der Natur der Sekundärdatennutzung, dass zum Zeitpunkt der Einwilligung nicht alle potenziellen Nutzungszwecke der Daten bekannt und absehbar sind. Wegen dieser unbestimmten zukünftigen Datennutzung werden in der medizinischen Forschung zunehmend Einwilligungen erbeten, die umfassender und allgemeiner formuliert sind, auch „Broad Consent“ genannt. Internationale Gremien der Gesundheitsforschung, darunter die World Medical Association und das Council for International Organizations of Medical Sciences/World Health Organization, haben die Umsetzung einer breiten Einwilligung als eine akzeptable Alternative gebilligt. 
Die MII hat sich mit dem sogenannten „Broad Consent“ bewusst für eine aktive informierte Einwilligung der Patientinnen und Patienten als Rechtsgrundlage für die Forschung mit Routinedaten der medizinischen Versorgung entschieden. Die Konferenz der unabhängigen Datenschutzbeauftragten des Bundes und der Länder hat am 15. April 2020 einem von der MII erarbeiteten bundesweit einheitlichen Mustertext für die Patienteneinwilligung zugestimmt. Auf diesen hatten sich alle an der MII beteiligten Universitätsklinikstandorte zuvor verständigt. Die Akzeptanz der Einwilligungsdokumente ermöglicht der medizinischen Forschung nun, auf Basis der EU-Datenschutzgrundverordnung (DSGVO) bundesweit eine breite Einwilligung in die Nutzung pseudonymisierter klinischer Daten einzuholen. Das ist eine wichtige Voraussetzung für eine Vielzahl von Forschungsvorhaben zur Optimierung der Patientenversorgung und der personalisierten Medizin bis hin zur Entwicklung von KI-basierten Entscheidungshilfen.

Mit dem „Broad Consent“ geben die Patientinnen und Patienten ihre informierte Einwilligung, dass ihre Gesundheitsdaten aus der Routineversorgung für einen Zeitraum von fünf Jahren in pseudonymisierter Form für die Forschung ausgewertet werden dürfen. Nach Ablauf dieser fünf Jahre muss erneut eine Einwilligung eingeholt werden. Wie das logistisch funktionieren kann und inwieweit das den Wünschen und Vorstellungen der Bürgerinnen und Bürger entspricht, ist bisher nicht geklärt. Studien im internationalen Raum haben gezeigt, dass über die Präferenzen hinsichtlich der Einwilligung der Betroffenen selbst wenig bekannt ist (Xafis et al. 2019, Howe et al. 2018).

Die Medizininformatik-Initiative schafft für die Öffentlichkeit außerdem ein Onlineportal, in dem sich Patientinnen und Patienten darüber informieren können, welche konkreten medizinischen Forschungsvorhaben mit den über diesen „Broad Consent“ verfügbar gemachten Daten durchgeführt werden (,,Transparenzportal“ der MII).

Um die Akzeptanz der deutschen Bevölkerung gegenüber einer Datenspende für die Forschung und die Präferenzen für verschiedene Formen der Einwilligung zu untersuchen, hat die Technologie- und Methodenplattform für die vernetzte medizinische Forschung (TMF e. V.) im August 2019 eine Befragung in der deutschen Bevölkerung durchgeführt, die wir im Folgenden näher vorstellen.

\section{Daten teilen für die Forschung - was die Deutschen denken}

Bürgerinnen und Bürger setzen sich zunehmend bewusster mit der Verwendung ihrer Daten auseinander. Deshalb ist es unumgänglich, die Bedürfnisse und Einstellungen der Öffentlichkeit zu berücksichtigen, wenn man über angemessene Formen der Einwilligung in die Sekundärdatennutzung für die medizinische Forschung diskutiert. Die Innovationsinitiative „Daten helfen heilen“ der Bundesregierung betont, dass es neuer Wege bedarf, ,um die Bereitschaft der Menschen, sich an Gesundheitsforschung $\mathrm{zu}$ beteiligen, $\mathrm{zu}$ unterstützen und $\mathrm{zu}$ fördern. Entscheidend wird dabei sein, dass 
Patientinnen und Patienten, Forschende und das Gesundheitspersonal den Daten verarbeitenden Stellen ein hohes Maß an Vertrauen entgegenbringen“ (Bundesministerium für Bildung und Forschung 2020).

Internationale Studien und Untersuchungen zeigen zunächst eine grundsätzlich positive Grundhaltung der Menschen gegenüber medizinischer Forschung, die mit einer hohen Bereitschaft einhergeht, medizinische Daten für die Forschung bereitzustellen (Kalkman et al. 2019, Garrison Nanibaa et al. 2016, Stockdale at al. 2018). Diese Bereitschaft ist jedoch meist an Bedingungen geknüpft. Die in den Studien am häufigsten genannten Gründe, medizinische Forschung zu unterstützen, sind zumeist altruistischer Natur (Nobile et al. 2016). Konkret genannt werden meist der Wille, zur Entwicklung besserer medizinischer Therapien beizutragen (Shabani et al. 2014, Mazor et al. 2017), zukünftige Patientengenerationen zu unterstützen oder der Wunsch, nach einer positiv erlebten Behandlung selbst etwas zurückgeben zu wollen (Richter et al. 2019). Letztendlich sind für viele befragte Menschen der Wunsch und die Hoffnung entscheidend, dass ihre Daten zu einer besseren Gesundheitsversorgung beitragen.

Durch die im Rahmen der Medizininformatik-Initiative durchgeführte Befragung sollte ein erstes Stimmungsbild in der deutschen Bevölkerung ermittelt werden, unter welchen Bedingungen die Bürger bereit wären, ihre Gesundheitsdaten mit der medizinischen Forschung zu teilen. Weiterhin sollte ermittelt werden, wem die Menschen ihre Gesundheitsdaten für Forschungszwecke zur Verfügung stellen würden und wie häufig die Bürger um Erlaubnis gefragt werden möchten.

Die bevölkerungsrepräsentative standardisierte Befragung wurde zwischen dem 13. bis 18. August 2019 vom Forschungsinstitut forsa Politik- und Sozialforschung GmbH im Auftrag der TMF - Technologie- und Methodenplattform für die vernetzte medizinische Forschung e. V. für die Medizininformatik-Initiative durchgeführt (TMF e. V. 2019). Befragt wurden 1.006 erwachsene deutsche Bürger ab 18 Jahren unter Verwendung des Online-repräsentativen Befragungspanels forsa.omninet mit einer Fehlertoleranz von + /-3 Prozentpunkten.

Die Umfrage umfasste vier Fragen:

(1) Einstellung zur Datenspende aus digitalen Gesundheitsakten,

(2) Gründe für die Ablehnung der Datenspende (offene Frage),

(3) mögliche Begünstigte der Datenspende und

(4) Dauer der Forschungsnutzung nach Datenspende.

\subsection{Großer Rückhalt gegenüber einer Datenspende für die Forschung}

Die Befragung der TMF e. V. zeigte, dass die Bürgerinnen und Bürger gegenüber der Verwendung ihrer digitalen Gesundheitsdaten für die medizinische Forschung grundsätzlich positiv eingestellt sind. Mehr als drei Viertel (79\%) der Deutschen wären bereit, 
ihre persönlichen Gesundheitsdaten für die Durchführung medizinischer Forschungsprojekte zur Verfügung zu stellen (Abb. 1).

In der positiven Grundhaltung gegenüber dem Teilen der eigenen Gesundheitsdaten gibt es keine signifikanten Unterschiede in den verschiedenen Altersgruppen und Regionen (Ost- und Westdeutschland). Männer waren gegenüber einer Datenspende mit $84 \%$ etwas positiver eingestellt als Frauen $(75 \%)$. Besonders überraschend war das Ergebnis, dass gesunde Menschen eine ebenso hohe Bereitschaft zum Datenteilen aufweisen wie chronisch Kranke (Abb. 2).

Ähnlich positive Ergebnisse hat eine Befragung der data4life-Initiative des HassoPlattner-Instituts zum Thema Forschungsunterstützung und Offenheit für die Datenspende im Frühjahr 2020 ergeben. Anlass der Befragung war die Corona-Pandemie. $73 \%$ der deutschen Bevölkerung könnte sich vorstellen, ihre Gesundheitsdaten für die Corona-Forschung digital zu spenden. Die Verbesserung der Therapie und Diagnose (63\%), die Vorbeugung von Krankheiten (57\%) und schnellere Erkenntnisse (54\%) waren die am häufigsten genannten Gründe für die Datenspende (data4life 2020).

Diejenigen Befragten, die eher nicht oder auf keinen Fall damit einverstanden wären, dass ihre persönlichen Gesundheitsdaten anonym und unentgeltlich für die medizinische Forschung zur Verfügung gestellt werden, wurden anschließend in einer offenen Frage nach den Gründen dafür gefragt. Mit Abstand am häufigsten (60\%) wird als Grund gegen eine solche Datenspende die Angst vor Datenmissbrauch bzw. vor der Nichteinhaltung des Datenschutzes genannt. Als weitere Gründe gegen die Zurverfügungstellung

\section{Einverständnis zur Datenspende für die medizinische Forschung}
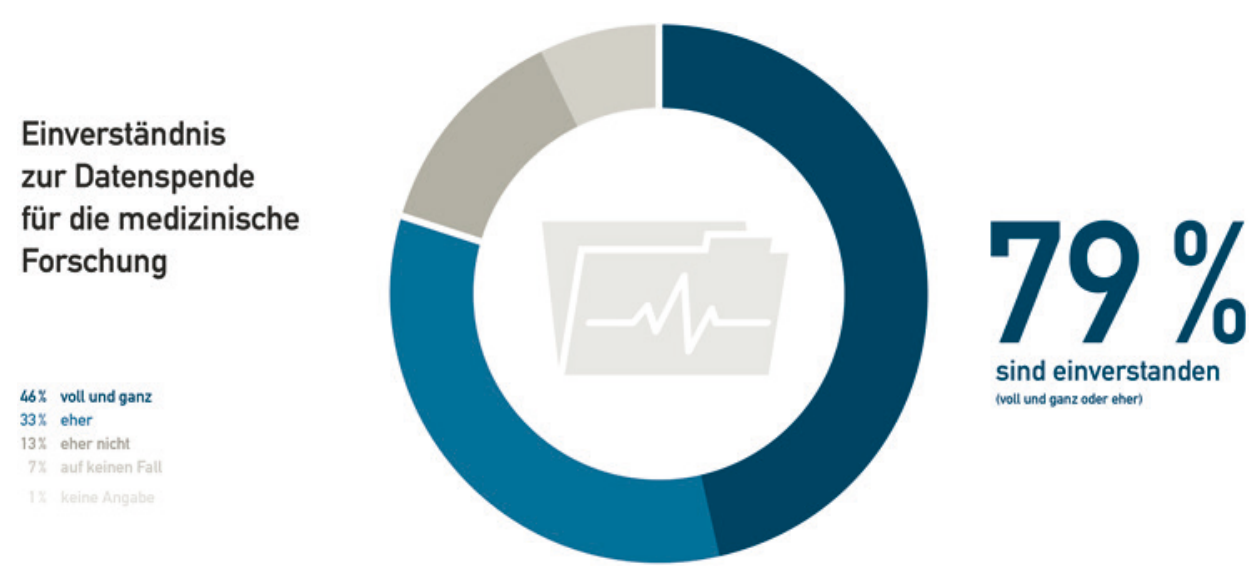
sind einverstanden (voll und ganz oder eher)

Abb. 1 Einverständnis zur Datenspende für die medizinische Forschung $(\mathrm{n}=1.006)$, Befragte ab 18 Jahren in Deutschland, Antwort auf die Frage: Einmal angenommen, in Zukunft würden Ihre persönlichen Gesundheitsdaten, wie z. B. Ihre Krankheitsgeschichte, Untersuchungsergebnisse, Röntgenbilder etc. in einer digitalen Gesundheitsakte online gespeichert werden. Wären Sie in diesem Falle einverstanden, dass Ihre persönlichen Gesundheitsdaten anonym und unentgeltlich für die medizinische Forschung zur Verfügung gestellt werden, damit zukünftig Krankheiten besser erkannt und neue Behandlungen entwickelt werden können? (Quelle: TMF e. V. 2019) 


\section{Einverständnis zur Datenspende für die medizinische Forschung}

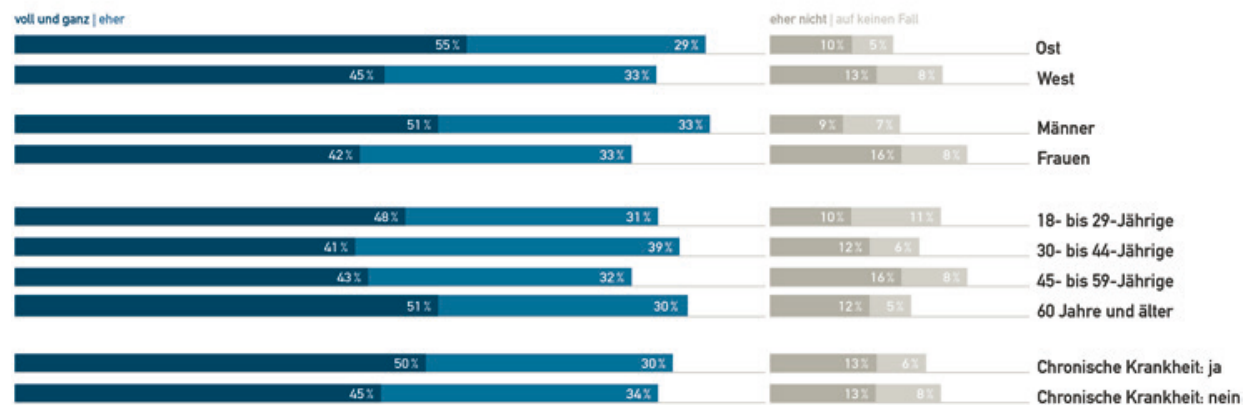

Abb. 2 Einverständnis zur Datenspende für die medizinische Forschung nach Region, Geschlecht, Alter, Erkrankungsstatus $(\mathrm{n}=1.006)$, Befragte ab 18 Jahren in Deutschland, Antwort auf die Frage: Einmal angenommen, in Zukunft würden Ihre persönlichen Gesundheitsdaten, wie z. B. Ihre Krankheitsgeschichte, Untersuchungsergebnisse, Röntgenbilder etc. in einer digitalen Gesundheitsakte online gespeichert werden. Wären Sie in diesem Falle einverstanden, dass Ihre persönlichen Gesundheitsdaten anonym und unentgeltlich für die medizinische Forschung zur Verfügung gestellt werden, damit zukünftig Krankheiten besser erkannt und neue Behandlungen entwickelt werden können? (Quelle: TMF e. V. 2019)

ihrer persönlichen Daten werden sowohl das Recht an den eigenen Daten (20\%) genannt, als auch der Nutzen ihrer persönlichen Gesundheitsdaten für die Forschung selbst (13\%) angezweifelt.

Die in der TMF-Befragung ermittelten Zustimmungswerte entsprechen ungefähr vergleichbaren Erhebungen in anderen Ländern. So erbrachte eine entsprechende Umfrage in der Bevölkerung der Niederlande eine Akzeptanzquote von $70.5 \%$ für die Nachnutzung von Gesundheitsdaten für die medizinische Forschung, dabei sogar 92,3\% mit ausdrücklicher Nutzung eines „Broad Consent“ (Richter et al. 2020). Eine etwas ältere repräsentative Umfrage in der Bevölkerung Kaliforniens zeigte Ähnliches: $76,2 \%$ der Befragten würden dort einer Nutzung ihrer Gesundheitsdaten für die Forschung zustimmen - obwohl mehrheitlich Risiken für die Privatheit und Sicherheit der Daten durch die Digitalisierung im Gesundheitswesen ganz allgemein gesehen werden. Interessanterweise ist hierbei die Zustimmungsquote zu einem Data Sharing im Forschungszusammenhang sogar größer als im Rahmen der Gesundheitsversorgung (76,2\% vs. 57,5\%), und eine sehr große Mehrheit wünscht hierfür explizit Einwilligungsbasiertheit $(95,5 \%)$ und Kontrollmöglichkeiten in Bezug auf die Datennutzung (Kim et al. 2015). 


\subsection{Patienten würden langfristige Datennutzung unterstützen}

Danach gefragt, wie bzw. wie lange ihre persönlichen Gesundheitsdaten nach ihrer Datenspende für die medizinische Forschung genutzt werden dürften, sprechen sich $73 \%$ der befragten Deutschen für eine langfristige Nutzung von mindestens fünf Jahren aus. $56 \%$ antworten, dass ihre Gesundheitsdaten zeitlich unbegrenzt für die medizinische Forschung genutzt werden dürften. Nur $27 \%$ wollen bei jedem einzelnen Forschungsprojekt nach ihrem Einverständnis gefragt werden (Abb. 3).

Die Ergebnisse lassen Rückschlüsse darauf zu, wie Ansätze und konkrete Modelle für ein an den Interessen und Bedürfnissen des Datenspenders orientiertes Einwilligungsund Nutzungsverfahren für medizinische Daten im Forschungskontext gestaltet werden könnten.

Beim Alter, der regionalen Herkunft und dem Gesundheitsstatus sind keine signifikanten Unterschiede im Antwortverhalten festzustellen. Bei den Männern war der Anteil derjenigen, die ihre Daten langfristig für die medizinische Forschung zur Verfügung stellen würden, etwas höher (79\%) als bei den Frauen (65\%) (Abb. 4).

\subsection{Befragte unterscheiden zwischen öffentlicher und kommerzieller Forschung}

$97 \%$ der befragten Personen, die damit einverstanden sind, dass ihre persönlichen Gesundheitsdaten für die medizinische Forschung genutzt werden, würden ihre Daten Wissenschaftlern an Universitäten und öffentlichen Forschungseinrichtungen zur Verfügung stellen. Hingegen würde nur eine Minderheit (17\%), darunter jüngere häufiger

Wie lange dürften die Gesundheitsdaten nach der Datenspende genutzt werden?
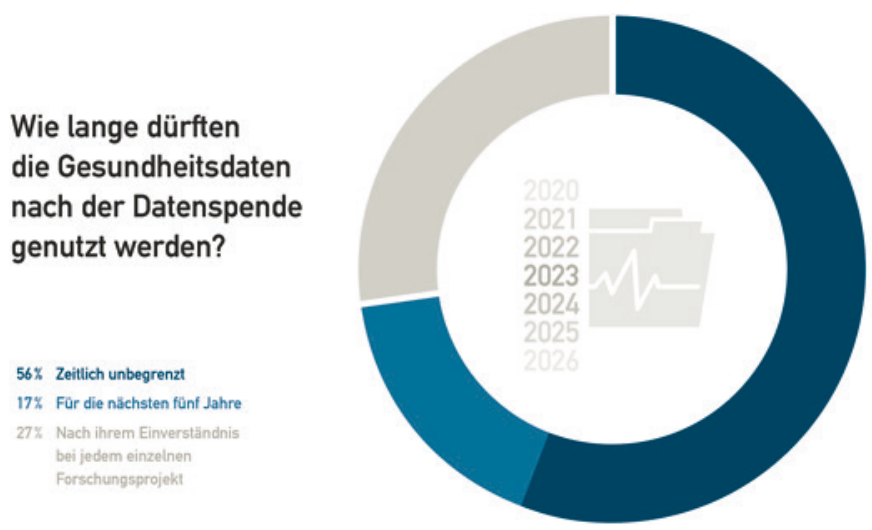

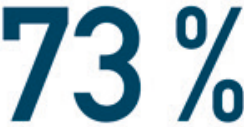

stimmen einer langfristigen Datennutzung zu (Zeitlich unbegrenzt und Nur die nächsten Nunt Jahre)

Abb. 3 Dauer der zeitlichen Nutzung der Gesundheitsdaten $(n=793)$, Antworten auf die Frage: Wie lange dürften Ihre persönlichen Gesundheitsdaten nach Ihrer Datenspende für die medizinische Forschung genutzt werden? (Quelle: TMF e. V. 2019) 
Wie lange dürften die Gesundheitsdaten nach der Datenspende genutzt werden?

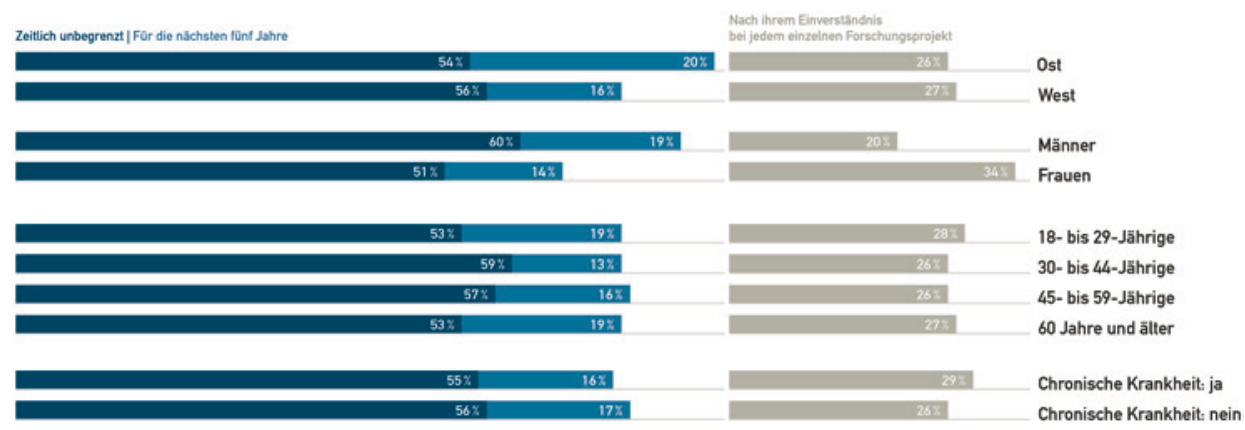

Abb. 4 Dauer der zeitlichen Nutzung der Gesundheitsdaten nach Region, Geschlecht, Alter, Erkrankungsstatus $(\mathrm{n}=793)$, Antworten auf die Frage: Wie lange dürften Ihre persönlichen Gesundheitsdaten nach Ihrer Datenspende für die medizinische Forschung genutzt werden? (Quelle: TMF e. V. 2019)

\section{Wem würden die Deutschen ihre Gesundheitsdaten zur Verfügung stellen? \\ 97\% Wissenschaftlern an Universitāten und offentlichen Forschungseinrichtungen \\ $17 \%$ Wissenschaftlern aus der Industrie und von privaten Unternehmen}

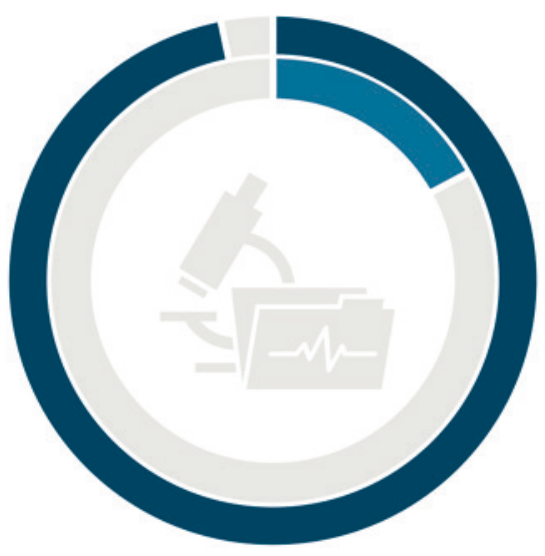

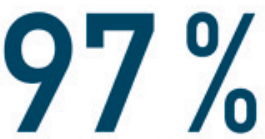

unterstützen öffentliche Forschung

Abb. 5 Nutzer einer Datenspende für die medizinische Forschung (Mehrfachnennung möglich) $(n=793)$, Antworten auf die Frage: Wem würden Sie Ihre persönlichen Gesundheitsdaten für die medizinische Forschung zur Verfügung stellen? (Quelle: TMF e. V. 2019)

als ältere Befragte, ihre Gesundheitsdaten mit Wissenschaftlern aus der Industrie und von privaten Unternehmen, wie z. B. Arzneimittelherstellern oder Biotechnologieunternehmen, teilen (Abb. 5).

Diese zurückhaltende Position gegenüber der Nutzung medizinischer Daten durch Forscher in kommerziellen Unternehmen findet sich auch in den Ergebnissen internationaler Befragungen wieder. Eine niederländische Patientenbefragung aus dem Jahr 2019 fragte Patienten nach ihrer Haltung gegenüber der Sekundärnutzung medizinischer Daten für andere als akademische Forschungszwecke wie z. B. die 
Arzneimittelentwicklung kommerzieller Organisationen. Während 28,8 \% der Befragten voll und ganz einverstanden wären, würden 42,3 \% ihre Erlaubnis vom Empfänger und dem Zweck der Datennutzung abhängig machen (Richter et al. 2020).

Eine amerikanische Studie des PwC Health Research Institute aus dem Jahr 2020, welche anlässlich der COVID-19 Pandemie durchgeführt wurde, deutet ebenfalls auf ein Vertrauensgefälle gegenüber Akademia und Industrie, selbst wenn es -wie in dieser Befragung und im Unterschied $\mathrm{zu}$ den beiden vorangegangen beschriebenen Befragungen mit unspezifischer Nutzung - um einen spezifischen Forschungszweck geht: $84 \%$ der Befragten würden mit ihrem eigenen Arzt Daten für die medizinische Forschung zu COVID-19 teilen, $75 \%$ mit einem lokalen Krankenhaus und lediglich $50 \%$ mit einem Arzneimittelhersteller (PwC Health Research Institute 2020).

Die beobachtete niedrig ausgeprägte Bereitschaft der Bürger, Daten mit der Arzneimittelindustrie zu teilen, stellt die Forschungslandschaft vor Herausforderungen. An der Entwicklung neuer Diagnostika oder Therapeutika sind zahlreiche Akteure aus dem privaten und öffentlichen Sektor beteiligt. Die meisten Studien werden in Kooperation zwischen nicht-kommerziellen und kommerziellen Einrichtungen durchgeführt. Die Befragungsdaten zeigen, dass hier Vermittlungsarbeit zu leisten ist, um Verständnis und Vertrauen beim Bürger zu schaffen.

\section{Schlussfolgerungen und Ausblick}

Digitale Gesundheitsdaten aus der Versorgung stellen eine wichtige Grundlage für Innovationen in der Medizin dar. Die systematische Nutzung dieser Daten, unter anderem für Big Data- und KI-Anwendungen im Sinne eines lernenden Gesundheitssystems, wird dazu beitragen, dass Therapien weiterentwickelt und Krankheiten besser und zielgerichteter im Sinne einer personalisierten Medizin geheilt werden können. Jedoch wirft die wachsende Vielfalt und Entgrenzung von Daten und die Möglichkeit ihrer De- und Rekontextualisierung für die medizinische Forschung viele regulatorische, ethische und soziale Fragen auf (Richter et al. 2020). Diese Fragen erfordern eine gesellschaftliche Auseinandersetzung mit der Zukunft der datenreichen Medizin, die sich nicht nur auf neue Einwilligungsmodelle oder Partizipationsformate beschränken lässt. Eine wichtige Voraussetzung für den Erfolg der datenreichen Medizin ist die Bereitschaft der Menschen, ihre Daten für die Forschung zur Verfügung zu stellen. Diese Bereitschaft hängt vom Vertrauen der Bevölkerung in die Institutionen, Personen und Prozesse ab, die die Gesundheitsdaten erheben, verarbeiten und auswerten (Bundesministerium für Bildung und Forschung 2020).

Je mehr digitale Gesundheitsdaten für die medizinische Forschung genutzt werden können, desto wirkungsvoller können diese Daten zur Verbesserung der Gesundheit der Bevölkerung beitragen. Gleichzeitig wird es für den einzelnen Bürger immer schwieriger, den Zugang zu seinen Daten zu überblicken und zu steuern. Angesichts dieser Entwicklung hat der Deutsche Ethikrat in seiner Stellungnahme „Big Data und 
Gesundheit“ aus dem Jahr 2017 das Konzept einer projektübergreifenden „Datenspende" für die medizinische Forschung eingeführt (Deutscher Ethikrat 2017). Eine „Datenspende” ist dabei zunächst einmal ein offener Begriff. Grundsätzlich setzt die Verarbeitung personenbezogener medizinischer Daten zu Forschungszwecken in der EU voraus, dass der Patient eingewilligt hat oder die Datennutzung gesetzlich wegen öffentlichen Forschungsinteresses erlaubt ist. Die rechtskonforme Nutzung bereits existierender, in der Regel für Versorgungszwecke erhobener, medizinischer Daten für Forschungszwecke ist grundsätzlich auf drei Arten denkbar: Erstens können die Patientinnen und Patienten in eine Datennutzung einwilligen. Zweitens kann der Gesetzgeber auf Grundlage von Artikel 9 Abs. 2j EU-Datenschutzgrundverordnung (DSGVO) eine Forschungsdatennutzung unabhängig vom Willen der Patientinnen und Patienten erlauben. Davon machen beispielsweise die Landeskrankenhausgesetze und einige Registergesetze, aber auch die Datenschutzgesetze Gebrauch. Darunter fällt auch die Forschung mit den Sozialdaten der Krankenkassen, die über das Datentransparenzverfahren des SGB V ausgewählten Nutzungsberechtigten zur Verfügung stehen. Drittens könnte man auch einen Mittelweg gehen, indem man auf eine gesetzliche Erlaubnis zurückgreift, aber trotzdem dem Betroffenen die Entscheidung überlässt - sei es, dass man ihn um seine Zustimmung fragt ("Opt-In”) oder ihm die Möglichkeit eines Widerspruchs gibt (“Opt-Out”) (Strech et al. 2020).

Bei jedweder Einwilligung mittels „Opt-In“ stellt sich im Rahmen herkömmlicher Verfahren zur Einwilligungseinholung als problematisch dar, dass die Einwilligung in die Datenspende während einer Behandlung im Krankenhaus im Kontext einer medizinischen Behandlung erfolgt. Neben den vielfach beschriebenen Problemen der „therapeutic misconception“ (Appelbaum et al. 2004) oder der „diagnostic misconception“ (Nobile et al. 2016) ist es ethisch problematisch, dass die Aufnahmefähigkeit der Datenspender in dieser Situation naturgemäß reduziert ist. Gleichermaßen steht das aufklärende klinische Personal in dieser Situation unter erheblichem Zeitdruck. Wie in der Literatur vielfach diskutiert wäre es empfehlenswert, die Entscheidung über die Datenspende vom klinischen Kontext, wenn nicht gar generell von der Situation des Erkranktseins zu trennen und im normalen Alltagsleben zu verankern.

Grundvoraussetzung für eine datenreiche Medizin ist ein hohes Niveau der Datensicherheit - das beginnt damit, dass es nur um pseudonymisierte Datenbestände geht, der Forscher also den Patienten nicht identifizieren kann. Ähnlich wichtig sind Vertrauen, Transparenz und Information. Die Bürger müssten über das Konzept der Datenspende informiert sein und einen Widerspruch jederzeit und leicht ausüben können. Hier wäre beispielsweise über ein nationales Patientenportal nachzudenken, das über Forschungsvorhaben informiert und wo ein Widerspruch hinterlegt werden könnte. Die Innovationsinitiative „Daten für Gesundheit“ kündigt in seiner Roadmap eine solche Informations- und Kommunikationsplattform für die datengestützte Gesundheitsforschung an, auf der Informationen zu Forschungsvorhaben, Datenspendemöglichkeiten und Ergebnisse vorgestellt werden sollen (Bundesministerium für Bildung und Forschung 2020). 
Trotz der großen Bereitschaft und der positiven Grundeinstellung der Bürgerinnen und Bürger, die Gesundheitsforschung mit Daten $\mathrm{zu}$ unterstützen, sind diese an Bedingungen geknüpft. Kalkman et al. haben die Rahmenbedingungen zusammengefasst in den Dimensionen ,value, privacy, minimising risks, data security, transparency, control, information, trust, responsibility and accountability“ (Kalkman et al. 2019). Die Dimensionen wurden auf Basis systematischer Reviews und Befragungen anderer Dateninitiativen ermittelt (Tab. 1).

Als Mittel zur Legitimation der Verwendung medizinischer Daten in der Sekundärforschung wird in Deutschland in der Medizininformatik-Initiative mit dem „Broad Consent" eine Politik verfolgt, die auf eine breite Einwilligung bei gleichzeitiger

Tab. 1 Bedingungen für das Teilen von Gesundheitsdaten nach Kalkman et al. 2019

\begin{tabular}{|c|c|}
\hline Value & $\begin{array}{l}\text { Forschung auf Basis gespendeter Daten sollte im } \\
\text { öffentlichen Interesse sein und die Werte der Datenspender } \\
\text { reflektieren. }\end{array}$ \\
\hline Privacy, risks, and data security & $\begin{array}{l}\text { Datenschutz, Wahrung der Privatsphäre und die } \\
\text { Minimierung von Risiken beim ,Data sharing ' müssen } \\
\text { oberste Prinzipien sein. }\end{array}$ \\
\hline Transparency and control & $\begin{array}{l}\text { Die Erwartung der Datenspender an Transparenz lässt sich } \\
\text { durch die Bereitstellung folgender Informationen erfüllen: } \\
\text { - die Art der Forschung, die durchgeführt werden soll, } \\
\text { - wer die Studie/das Forschungsprojekt durchführt, } \\
\text { - Informationen darüber, wie und mit wem Daten aus- } \\
\text { getauscht werden, } \\
\text { - Informationen über die Governance der datenverwaltenden } \\
\text { Stelle und der Sicherheitsvorkehrungen, sowie zur Daten- } \\
\text { verwaltung, } \\
\text { - Bedingungen für den Datenzugang, } \\
\text { - Transparenz über Partnerschaften mit der Industrie, } \\
\text { - Information der Datenspender über die Datennutzung und } \\
\text { die Studienergebnisse, } \\
\text { - Möglichkeiten der Kontrolle und des Widerspruchs seitens } \\
\text { der Datenspender }\end{array}$ \\
\hline Information and trust & $\begin{array}{l}\text { Patientenedukation und Informationen, unter anderem in } \\
\text { Form öffentlicher Kampagnen, müssen sicherstellen, dass } \\
\text { Datenspender ausreichend verstehen, was mit ihren Daten } \\
\text { passiert und wie diese verwendet werden. } \\
\text { Vertrauen wird vor allem der primären Kontaktinstitution } \\
\text { entgegengebracht. }\end{array}$ \\
\hline Responsibility and accountability & $\begin{array}{l}\text { Unabhängige Use and Access Committees, bestehend } \\
\text { aus Vertretern aller Stakeholdergruppen, sollten über die } \\
\text { Sekundärdatennutzung entscheiden. } \\
\text { Sanktionen, Strafen und Konsequenzen bei Datenmiss- } \\
\text { brauch müssen transparent und klar geregelt sein. }\end{array}$ \\
\hline
\end{tabular}


einheitlich reglementierter Kontrolle der Datennutzungen durch unabhängige Dritte (Ethikkommissionen, Use \& Access Committees) setzt. Allerdings erfordert die Schaffung immer komplexerer Infrastrukturen für die Datenintegration aus unterschiedlichen Quellen perspektivisch eine Weiterentwicklung der Einwilligungsverfahren. Obwohl es Einigkeit über die Voraussetzungen für einen verantwortungsvollen und damit ethisch akzeptablen Datenaustausch zu geben scheint, wie dem Schutz der Privatsphäre, der Risikominimierung, der Datensicherheit, der Transparenz sowie der Information und des Vertrauens der Öffentlichkeit, ist wenig über die konkrete Haltung der betroffenen Bürgerinnen und Bürger selbst bekannt.

Die Umfrage der TMF e. V. liefert erste Einblicke in die Haltung der betroffenen Öffentlichkeit in Deutschland auf dem Weg zu einer umfassenden Nutzung der Sekundärforschung ihrer Daten im Rahmen einer „Opt-Out“-Politik („Datenspende“). Zwei Ergebnisse dieser Umfrage sind wesentlich für die Diskussion um die Ausgestaltung und Form der Datenspende: Neben der allgemeinen breiten Bereitschaft der Bevölkerung, die Daten in Form einer Datenspende der medizinischen Forschung zur Verfügung zu stellen, ist die Haltung gegenüber zukünftigen Nutzern wesentlich für die weitere Diskussion. Während Universitäten und öffentliche Forschungseinrichtungen großes Vertrauen genießen, wird die Nutzung der Daten für die kommerzielle Forschung kritischer gesehen. Diese Ressentiments gegenüber der wissenschaftlichen Nutzung der gespendeten Daten durch kommerzielle Institutionen sind nicht nur im Forschungsalltag schwer zu berücksichtigen, wo die Zusammenarbeit zwischen öffentlich finanzierter und kommerzieller Forschung längst Realität ist. Vielmehr wird dies mittlerweile auch von vielen Finanzierungsorganisationen gefördert. Gerade das Beispiel der häufig aus dem akademischen Umfeld ausgegründeten Start-Ups macht deutlich, wie schwierig die Grenze zu ziehen ist. Medikamente, Impfstoffe oder personalisierte Therapien werden schon heute nur in enger Kooperation zwischen öffentlich geförderter Grundlagenforschung, privatwirtschaftlicher Produktentwicklung und akademischer klinischer Forschung entwickelt werden.

Das Versprechen der datengetriebenen medizinischen Innovation braucht eine Kultur des Datenteilens. Voraussetzung hierfür ist ein gesellschaftlicher Diskurs, in den neben den Stakeholdern aus Wissenschaft und Industrie auch die betroffenen Patientinnen und Patienten ihre Ansprüche an Transparenz, Kontrolle und Nutzen der geplanten Datennutzung einbringen können. Im Zentrum muss die Datenhoheit der Bürgerinnen und Bürger stehen, die das letzte Wort darüber haben, in welcher Form ihre Daten für heutige und zukünftige Forschungsfragen verwendet werden dürfen.

\section{Förderhinweis}

Diese Arbeit ist im Rahmen des Projekts „Medizininformatik-Initiative - Teilprojekt Koordinationsstelle der Medizininformatik-Initiative“ entstanden. Dieses wird unter dem Förderkennzeichen 01ZZ1805 durch das Bundesministerium für Bildung und Forschung gefördert. 


\section{Literatur}

Appelbaum PS, Lidz CW, Grisso T (2004) Therapeutic misconception in clinical research: frequency and risk factors. IRB 26(2):1-8. PMID: 15069970. https://doi.org/10.1016/s02779536(03)00338-1

Australian Government, Office of the Australian Information Commissioner (2020) My Health Record. https://www.oaic.gov.au/privacy/other-legislation/my-health-record/. Zugegriffen: 21. Okt. 2020

Bundesministerium für Bildung und Forschung (2020) Innovationsinitiative "Daten für Gesundheit" Roadmap für eine bessere Patientenversorgung durch Gesundheitsforschung und Digitalisierung. https://www.bmbf.de/de/daten-helfen-heilen-12503.html. Zugegriffen: 23. Okt. 2020

Data4life (2020) Pulscheck Deutschland zu COVID-19 und Datenspende. https://www.data4life. care/de/corona/umfrage/\#survey. Zugegriffen: 21. Okt. 2020

Deloitte (2016) International review Secondary use of health and social care data and applicable legislation. Available via SITRA. https://www.sitra.fi/en/publications/international-reviewsecondary-use-health-and-social-care-data-and-applicable/. Zugegriffen: 23. Okt. 2020

Deutscher Ethikrat (2017) Big Data und Gesundheit - Datensouveränität als informationelle Freiheitsgestaltung. Stellungnahme. Available via Deutscher Ethikrat. https://www.ethikrat.org/ fileadmin/Publikationen/Stellungnahmen/deutsch/stellungnahme-big-data-und-gesundheit.pdf. Zugegriffen: 28. Juli 2020

Europäische Kommission (2020) Eine europäische Datenstrategie. Mitteilung der Kommission an das europäische Parlament, den Rat, den europäischen Wirtschafts- und Sozialausschuss und den Ausschuss der Regionen. COM(2020) 66 final, 19.02.2020. https://ec.europa.eu/info/sites/ info/files/communication-european-strategy-data-19feb2020_de.pdf

Garrison Nanibaa A, Sathe NA, Antommaria AHM et al (2016) A systematic literature review of individuals' perspectives on broad consent and data sharing in the United States. Genet Med 18(7):663-671. https://doi.org/10.1038/gim.2015.138 Epub 2015 Nov 19

Howe N, Giles E, Newbury-Birch D, McColl E (2018) Systematic review of participants' attitudes towards data sharing: a thematic synthesis. J Health Serv Res Policy 23(2):123-133. https://doi. org/10.1177/1355819617751555

Kalkman S, van Delden J, Banerjee A et al (2019) Patients' and public views and attitudes towards the sharing of health data for research: a narrative review of the empirical evidence. J Med Ethics. pii: medethics-2019-105651. https://doi.org/10.1136/medethics-2019-105651

Kim KK, Joseph JG, Ohno-Machado L (2015) Comparison of consumers' views on electronic data sharing for healthcare and research. J Am Med Inform Assoc 22:821-830. https://doi. org/10.1093/jamia/ocv014

Mazor KM, Richards A, Gallagher M et al (2017) Stakeholders' views on data sharing in multicenter studies. J Comp Eff Res 6(6):537-547. https://doi.org/10.2217/cer-2017-0009 Epub 2017 Aug 14

Medizininformatik-Initiative (2020) Über die Initiative. https://www.medizininformatik-initiative. de/de/ueber-die-initiative. Zugegriffen: 22. Juli 2020

Ministry of Social Affairs and Health (2019) New act enables effective and secure use of health and social data. https://stm.fi/en/artikkeli/-/asset_publisher/uusi-laki-mahdollistaa-sosiaali-jaterveystietojen-tehokkaan-ja-tietoturvallisen-kayton. Zugegriffen: 1. Juli 2020

Nobile H et al (2016) Participants' accounts on their decision to join a Cohort Study with an attached biobank: a qualitative content analysis study within two german studies. J Empir Res Hum Res Ethics 11(3):237-249. https://doi.org/10.1177/1556264616657463

Parikka H (2019) A finnish model for the secure and effective use of data. Lessons learned from Sitra's Isaacus project. Available via SITRA. https://www.sitra.fi/en/publications/a-finnishmodel-for-the-secure-and-effective-use-of-data/ Zugegriffen: 23. Okt. 2020 
PwC Health Research Institute (2020) The COVID-19 pandemic is influencing consumer health behavior. Are the changes here to stay? https://www.pwc.com/us/en/library/covid-19/covid-19consumer-behavior.html. Zugegriffen: 23. Okt. 2020

Richter G, Borzikowsky C, Lieb W, Schreiber S, Krawczak M, Buyx A (2019) Patient views on research use of clinical data without consent: legal, but also acceptable? Eur J Hum Genet 27:841-847. https://doi.org/10.1038/s41431-019-0340-6

Richter G, Borzikowsky C, Lesch W, Semler SC, Bunnik EM, Buyx A, Krawczak M (2020) Secondary research use of personal medical data: attitudes from patient and population surveys in The Netherlands and Germany. Eur J Hum Genet. 2020 Oct 1. Online ahead of print. PMID: 33005018 https://doi.org/10.1038/s41431-020-00735-3

Semler SC, Wissing F, Heyder R (2018) German Medical Informatics Initiative - a national approach to integrating health data from patient care and medical research. Methods Inf Med 57(S 01):e50-e56. https://doi.org/10.3414/me18-03-0003

Shabani M, Bezuidenhout L, Borry P (2014) Attitudes of research participants and the general public towards genomic data sharing: a systematic literature review. Expert Rev Mol Diagn 14(8):1053-1065. https://doi.org/10.1586/14737159.2014.961917 Epub 2014 Sep 26

Stockdale J, Cassell J, Ford E (2018) "Giving something back": A systematic review and ethical enquiry into public views on the use of patient data for research in the United Kingdom and the Republic of Ireland. Wellcome Open Res 17(3):6. https://doi.org/10.12688/ wellcomeopenres.13531.2. eCollection 2018

Strech D, Graf von Kielmansegg S, Zenker S, Krawczak M, Semler SC (2020) Datenspende Bedarf für die Forschung, ethische Bewertung, rechtliche, informationstechnologische und organisatorische Rahmenbedingungen. Wissenschaftliches Gutachten für das BMG. TMF e.V. 2020. Zugänglich via Bundesministerium für Gesundheit. https://www.bundesgesundheitsministerium.de/en/service/publikationen/ministerium/details.html?bmg\%5Bpubid\%5D $=3424$. Zugegriffen: 20. Okt. 2020

TMF e. V. (2019) Deutliche Mehrheit der Deutschen bereit zur Datenspende für die medizinische Forschung. http://www.tmf-ev.de/News/articleType/ArticleView/articleId/4456.aspx. Zugegriffen: 20. Okt. 2020

Wilkinson MD, Dumontier M, Aalbersberg IJ et al (2016) The FAIR Guiding Principles for scientific data management and stewardship. Sci Data 3:160018. https://doi.org/10.1038/ sdata.2016.18

Xafis V, Schaefer GO, Labude MK et al (2019) An ethics framework for big data in health and research. ABR 11:227-254. https://doi.org/10.1007/s41649-019-00099-x

Open Access Dieses Kapitel wird unter der Creative Commons Namensnennung 4.0 International Lizenz (http://creativecommons.org/licenses/by/4.0/deed.de) veröffentlicht, welche die Nutzung, Vervielfältigung, Bearbeitung, Verbreitung und Wiedergabe in jeglichem Medium und Format erlaubt, sofern Sie den/die ursprünglichen Autor(en) und die Quelle ordnungsgemäß nennen, einen Link zur Creative Commons Lizenz beifügen und angeben, ob Änderungen vorgenommen wurden.

Die in diesem Kapitel enthaltenen Bilder und sonstiges Drittmaterial unterliegen ebenfalls der genannten Creative Commons Lizenz, sofern sich aus der Abbildungslegende nichts anderes ergibt. Sofern das betreffende Material nicht unter der genannten Creative Commons Lizenz steht und die betreffende Handlung nicht nach gesetzlichen Vorschriften erlaubt ist, ist für die oben aufgeführten Weiterverwendungen des Materials die Einwilligung des jeweiligen Rechteinhabers einzuholen. 